\title{
On the locus of visual dominance
}

\author{
HOWARD E. EGETH and LAWRENCE C. SAGER \\ The Johns Hopkins University, Baltimore, Maryland 21218
}

\begin{abstract}
A series of six experiments explored the dominance of vision over audition reported by Colavita (1974). We first confirmed the existence of visual dominance in a paradigm somewhat different from Colavita's: Mean reaction time (RT) to a light was found to be faster than to a simultaneously presented tone, even though the stimuli were equated in subjective intensity and even though RT to the tone presented alone was faster than to the light presented alone. Additional experiments showed that when subjects did not have to respond to light, tone RT was equal or faster (intersensory facilitation) when a light was present than when it was not. These findings suggest that sensory or perceptual processing of the tone is not affected by the light, i.e., that visual dominance is nonsensory in locus and depends on the relevance of the light stimulus. This interpretation was reinforced by other findings which showed that the degree of visual dominance was sensitive to the probability of light, tone, and light-plus-tone trials and to instructions to attend to a specific modality, but was not sensitive to the intensity of the light.
\end{abstract}

Can we hear and see at the same time? Although casual introspection suggests that the answer to this question is clearly "yes," if we turn to the psychological literature, the answer is much less clear. The question is essentially one about the division of attention, i.e., about the ability to process two or more sources of information independently. On the one hand, there is evidence that simultaneously presented stimuli, such as a tone and a light, may be processed independently or nearly independently (e.g., Eijkman \& Vendrik, 1965; Moore \& Massaro, 1973; Tulving \& Lindsay, 1967). On the other hand, there is evidence that processing two or more stimuli or aspects of one stimulus may result in a performance decrement (e.g., Egeth \& Pachella, 1969; Gilliom \& Sorkin, 1974; Lindsay, 1970; Massaro \& Kahn, 1973).

One of the most striking instances of a performance decrement in processing two simultaneous stimuli is Colavita's (1974) demonstration of what he called "sensory dominance." Subjects were presented with a series of suprathreshold stimuli consisting of a tone and a light in random alternation. They had two response keys, one for the tone and one for the light. Thus the experimental sequence was much like that of a conventional choice reaction time (RT) task with two stimuli and two responses. However, on occasional trials, both stimuli were presented simultaneously. When this happened, although the sub-

This research was supported in part by a contract between Johns Hopkins University and the Engineering Psychology Programs, Office of Naval Research, and in part by a grant from the National Science Foundation (BNS-76-01227) to Howard Egeth and James Pomerantz. The authors would like to thank Alfonso Caramazza, John Jonides, Raymond Nickerson, James Pomerantz, and William Stark for helpful advice. jects could (and should) have pressed both keys, they, in fact, usually pressed only the light key. Moreover, they sometimes seemed to be totally unaware that the tone had also been presented. This was the case despite the fact that the tone and light were equated in subjective intensity, and despite the fact that, on preliminary control trials using a simple RT paradigm, the tone was responded to more quickly than the light.

This demonstration is interesting and potentially important for a number of reasons. (1) Unlike most studies of multisensory stimulation, stimuli were far above threshold. Independence may be the rule with weak signals, interaction with strong signals. (2) Even though the auditory and visual signals were far above threshold, on dual stimulation trials subjects were sometimes totally unaware that a tone was presented. This occurred even in a condition in which the tone had twice the subjective intensity of the light. (3) Unlike many studies of divided attention, a strong asymmetry between vision and audition was observed. (For a more complete discussion of other instances of visual dominance in normal subjects, see Posner, Nissen, and Klein, 1976.) (4) The dominance of vision over audition in this study using normal adult subjects seems to be analogous to a phenomenon called "extinction" that has been known to neurologists for almost a century (for a review, see Bender, 1970). Extinction refers to the abnormal inhibition of one sensory experience by another. It can be either intramodal or intermodal, and is found most frequently in patients with cerebral lesions, in young children, and in very old people.

Our aim in this project was, first, to verify the existence of visual dominance as described by Colavita. Succeeding in this, we next tried to deter- 
mine the locus of the effect, i.e., whether it is actually a sensory phenomenon such as some form of masking, as opposed to, say, a decisional or response phenomenon. (Examples of what we would consider to be intersensory interactions are discussed by Melzack and Wall, 1965, in connection with their gate-control theory of pain.) Our logic in this effort was to assume that if the dominance occurred at an early, sensory stage of processing, it would be unaffected by manipulations (e.g., of response mode) that presumably affect later, more cognitive stages.

\section{EXPERIMENT 1}

\section{Method}

Our purpose in this experiment was to see if we could obtain evidence of sensory dominance under conditions similar to Colavita's.

Ten college students, six male and four female, served as paid subjects. Each was run individually in a session lasting about $20 \mathrm{~min}$.

The visual stimulus was presented in a conventional mirrortachistoscope. It consisted of the illumination of a white circle, $1.6^{\circ}$ in diameter, centered in the visual field at a viewing distance of $91 \mathrm{~cm}$. The visual field was dark between trials. The auditory stimulus was a $4,000-\mathrm{Hz}, 65 \mathrm{~dB}$ SPL tone, which was presented binaurally over earphones.

The subjects were run in a darkened room. After moderate dark adaptation, each subject matched the light and the tone for subjective intensity. The experimenter adjusted the intensity of the light until the subject reported that it was as bright as the tone was loud. The average of the three "ascending" and three "descending" trials was taken as the point of subjective equality. These matched stimuli were then used in the subsequent reaction time tasks.

Two response keys were located on a table directly in front of the subject. One was designated as the "tone" key and the other was called the "light" key. The tone key was on the right for five subjects (four of whom were right-handed), and the light key was on the right for the other five subjects (four of whom were righthanded). The subjects were instructed to press the tone key as soon as they heard the tone, or the light key as soon as they saw the light. A keypress terminated the corresponding stimulus. Light and tone onsets were controlled manually by the experimenter. Reaction times were measured to the nearest millisecond. After four practice trials, each subject was given 30 "simple" RT trials. On each of these trials, the subject was told whether a light or tone would occur. This verbal instruction was followed by an auditory warning click which preceded the stimulus by $500 \mathrm{msec}$. The intertrial interval was approximately $5 \mathrm{sec}$.

Following the simple RT trials, the subjects received 30 "choice" trials on which they did not know which stimulus would appear. Fifteen light and 15 tone trials were presented in a random sequence. Randomly interspersed among these 30 trials were five additional trials (called dual stimulation trials) on which the light and tone stimuli occurred simultaneously. As before, the subjects were instructed to press the tone key when the tone occurred and the light key when the light occurred. They were also told that both stimuli would be presented on a few trials and they were explicitly instructed to press both keys when this occurred. However, they were not given any instruction about the order in which the keys were to be pressed.' Each trial was preceded by a verbal "ready" signal which cooccurred with a click that indicated that a stimulus would appear in $500 \mathrm{msec}$.

\section{Results}

Reaction time. The mean simple RTs for tone and light trials were 288 and $320 \mathrm{msec}$, respectively, $\mathrm{t}(9)=2.62, \mathrm{p}<.05$. The mean RTs in the choice task were 427 and $429 \mathrm{msec}$ for tone and light, respectively, $t(9)<1$. This replicates the pattern of results found by Colavita for simple and choice trials.

On dual trials, the light response was made first on 34 of the 50 trials. Nine of the 10 subjects made the light response first on a majority (i.e., at least 3 out of 5) of their dual trials, $p=.022$ by sign test. (The data for the one discrepant subject actually resulted in a tie; they did not demonstrate tonal dominance.)

On dual trials, when light was responded to first, mean light RT was $419 \mathrm{msec}$. When tone was responded to first, mean tone RT was $506 \mathrm{msec}$. While this difference is consistent with the notion of visual dominance over audition, the effect was not significant $(p>.10) .^{2}$

These data support Colavita's finding that on dual stimulation trials a visual stimulus dominates an auditory stimulus, even though the stimuli were matched for subjective intensity and even though the tone results in faster responses than the light on simple RT trials. ${ }^{3}$

Errors. There were too few errors to support any meaningful discussion (only one in the entire experiment).

\section{EXPERIMENT 2}

In our first experiment, visual dominance was manifested in a tendency to respond to the light before the tone. In Colavita's experiments, the subjects were not explicitly instructed to push both keys on dual trials, and dominance was manifested in a tendency to respond to light but not to tone. Still another measure of visual dominance can be constructed if subjects are instructed to ignore the light and respond only to tones. We may then compare tone RT on choice and dual trials; to the extent that perceptual processing of the tone is interfered with by simultaneous presentation of light, dual trials ought to be slower than choice trials.

\section{Method}

Sixteen college students, eight male and eight female, served as paid subjects. The apparatus and stimuli were identical to that of Experiment 1, except that the stimuli were $50 \mathrm{msec}$ in duration.

The procedure was identical to that of Experiment 1 up through the simple RT task. Following the simple RT trials, the subjects were told that they would receive a random sequence of 35 trials (15 tone, 15 light, and 5 dual, i.e., tone plus light) and that they should press the tone key whenever they heard a tone. Thus this phase of the experiment required a kind of Donders' (1868) c-reaction. 


\section{Results}

Reaction time. The mean simple RTs for tone and light trials were 272 and $305 \mathrm{msec}$, respectively, $t(15)=2.74, p<.02$. Once again, we have demonstrated that, for subjectively equated stimuli, simple auditory RT is faster than simple visual RT.

In the next phase of the experiment, mean RT to tone alone was $347 \mathrm{msec}$ and to tone when accompanied by light $375 \mathrm{msec}, \mathrm{t}(15)=1.44, \mathrm{p}>.10$. Only 10 of the 16 subjects had faster mean responses to tone alone than to tone plus light, $p=.454$ by sign test.

Errors. There were too few errors to support any meaningful discussion (only two in the entire experiment).

\section{EXPERIMENT 3}

Our tentative conclusion from Experiment 2 was that visual dominance was not a sensory phenomenon, such as masking, since it was eliminated by a change in response mode. One subject (in Experiment 1) seemed to capture the essence of the phenomenon when he reported that, on dual trials, "I could see the light and hear the tone, but I couldn't push both switches together."

However, our conclusions are tentative for several reasons. First, the difference in RT between the tone and tone-plus-light trials, while statistically nonsignificant, was of substantial magnitude $(28 \mathrm{msec})$. Perhaps the difference was insignificant because so few trials entered into the calculation of each subject's mean. In the present experiment, each subject received 300 trials instead of just 35 .

Second, our results stand in conflict with some findings in the literature on intersensory facilitation of reaction time (see Nickerson, 1973, for a review). It has been found that reaction time to a signal can be speeded up by the simultaneous or near simultaneous presentation of an uninformative accessory stimulus. Typically, a tonal accessory stimulus improves RT to a light more than a visual accessory improves $\mathrm{RT}$ to a tone, but some slight effects can be expected even in the latter circumstances (e.g., Bernstein, Chu, Briggs, \& Schurman, 1973). Note that, although there was no significant effect of the visual accessory stimulus in our experiment, the 28 -msec effect that was observed is in the direction opposite to what would be expected on the basis of intersensory facilitation.

It is not clear why our experimental results differed from those of the formally similar intersensory facilitation experiments. We have already pointed out that the small number of trials in our experiment may have produced unstable results. Our search of the literature also suggested that, in the typical intersensory facilitation experiment, the proportion of trials containing an accessory in addition to the primary stimulus is much higher than the proportion of dual trials in our experiment (5 out of 35 trials $=.14)$. Although it was not obvious why this factor should influence the degree of interference or facilitation, in the absence of systematic data we felt it worth exploring. Consequently, we used two levels of probability of dual stimulation in the present experiment.

Since we were using an extended number of trials and were manipulating probability of dual stimulation trials, it seemed worthwhile to take this opportunity to compare under these conditions the creaction of Experiment 2 with a response that had shown strong interference in Colavita's paper. The most appropriate comparison seemed to be with the procedure of his Experiment IV, in which subjects were informed of the existence of dual stimulation trials and were explicitly instructed to respond to light and tone as appropriate on choice trials, but to press just the tone key when light and tone occurred simultaneously on dual trials.

\section{Method}

Forty college students, 20 male and 20 female, served as paid subjects. They were divided into four groups with nearly equal numbers of males and females in each group.

The apparatus and stimuli were the same as in Experiment 2. Each subject first matched the tone and light for subjective intensity. No simple RT trials were conducted. There was no verbal "ready" signal, but the subjects still heard a warning click $500 \mathrm{msec}$ before the stimulus. The intertrial interval was reduced to about $3 \mathrm{sec}$.

The four groups formed a 2 by 2 factorial design. One factor was Response Type. Two groups were instructed to use the Donders' c-reaction of Experiment 2: respond when and only when a tone is present. The other two groups were instructed to use the instruction used by Colavita (Experiment IV): respond to tone and light as appropriate when either is presented alone, but when both occur together, respond only to tone. For the sake of simplicity, we shall refer to these as, respectively, one- and two-key tasks. To ensure that subjects in the one-key conditions were not, either literally or figuratively, closing their eyes, they were warned that after occasional trials they would be asked whether or not a light had just been presented. There were no errors in response to these probes. The other factor was probability of dual stimula tion trials; two groups served under high probability, two under low probability. High probability refers to the following distribution of conditions over 300 test trials: 60 light, 120 tone, 120 dual $(.2, .4, .4)$. Low probability refers to the following distribution: 120 light, 120 tone, 60 dual, $(.4, .4, .2) .{ }^{4}$ The four conditions are illustrated in Table 1 .

Each subject received 10 practice trials, which were immediately followed by the 300 test trials. The session lasted about $35 \mathrm{~min}$.

\section{Results and Discussion}

Reaction time. As in Experiment 2, the measure of visual dominance (i.e., inhibition) or facilitation is obtained by subtracting the RT on dual trials from the RT on choice trials. Thus a positive difference score indicates intersensory facilitation and a negative difference score indicates intersensory inhibition, i.e., visual dominance over audition. The mean difference score for each condition is shown in 
Table 1. A two-way analysis of variance was carried out on these differences. The main effect of response type was significant, $F(1,36)=18.22, p<.001$. This finding reflects a 19-msec facilitation effect for the one-key response vs. a $20-\mathrm{msec}$ interference effect for the two-key response. The main effect of probability of dual stimulation was also significant, $F(1,36)=29.74, p<.001$. This finding reflects the 24-msec facilitation effect found in the high probability condition vs. the 25 -msec interference effect found in the low probability condition. The interaction of probability and response type was significant, $F(1,36)=8.56, p<.01$.

Tests of simple main effects showed that the effect of probability of dual trials was significant in the two-key condition $(-57$ vs. $+18 \mathrm{msec}), F(1,36)=$ $35.10, \mathrm{p}<.001$, but was not significant in the onekey condition $(+8 \mathrm{vs}$. $+30 \mathrm{msec}), \mathrm{F}(1,36)=3.20$, $\mathrm{p}>.05$. Thus, intersensory facilitation as classically measured by one-key tasks seems not to be greatly affected by probability of dual stimulation, whereas the "dominance" effect studied by Colavita is strongly dependent on that probability and can be reversed under appropriate circumstances. We should note that these data suggest that the nonsignificant reversal of intersensory facilitation found in Experiment 2 is just that-a nonsignificant effect, based on a small number of trials, that should not be taken seriously.

Note that the low-probability conditions of Experiment 3 replicate the essential findings of both our Experiment 2 and Colavita's Experiment IV, i.e., a nonsignificant effect with the one-key Donders' c-reaction but strong interference using Colavita's two-key response. (Probability of dual stimulation was .20 in the relevant conditions of the present study vs. .14 for our Experiment 2 and .17 for Colavita's Experiment IV.)

We have interpreted the fact that visual dominance disappeared in the one-key condition to mean that the overshadowing of tone by light in the two-key condition is not a sensory phenomenon. Without attempting a definition of the term sensory, we should say that we have implicitly taken it to refer to "hard-wired" processing. As an example of the kind of process we have in mind, we would point to lateral inhibition (see, e.g., Békésy, 1968; Cornsweet, 1970). There is little evidence that such inhibition is affected by cognitive factors such as instructional set or response mode. However, one still might wish to claim that results from our one-key condition are essentially irrelevant to the sensory dominance found in the two-key conditions, because subjects could, if they wished, ignore the irrelevant visual input. We think this is not a reasonable point of view. The subjects were warned that they would be asked occasionally whether or not a light had appeared on the preceding trial. They performed with perfect accuracy on these probe trials. There is also internal evidence that the subjects were not tuning out the visual input at a low level. We refer to the significant facilitation by light of tone RT in the one-key high probability condition. Obviously, if light input were being filtered out at a sensory level, it could not have caused any such facilitation.

Errors. Percentages of erroneous responses for light, tone, and dual trials appear in Table 1.

In the one-key conditions, errors occurred when subjects falsely responded to the light by pressing the tone key (there were no errors of omission in this or any other condition reported in this paper). In the high-probability-of-dual-stimulation condition, the false alarm rate was $9.3 \%$, and in the low-probabilityof-dual-stimulation condition, the false alarm rate was $1.7 \%, t(18)=2.85, p<.02$. In the former condition, tone responses were expected on $80 \%$ of the trials, while in the latter condition, they were expected on $60 \%$ of the trials. Thus, the error data suggest a criterion shift sensitive to the overall probability of having to respond to tone (or alternatively

Table 1

Design and Results of Experiment 3

\begin{tabular}{|c|c|c|c|c|c|c|c|c|}
\hline & \multicolumn{4}{|c|}{ High Probability of Dual Stimulation } & \multicolumn{4}{|c|}{ Low Probability of Dual Stimulation } \\
\hline & Light & Tone & Dual & $\begin{array}{l}\text { Inhibition or } \\
\text { Facilitation }\end{array}$ & Light & Tone & Dual & $\begin{array}{l}\text { Inhibition or } \\
\text { Facilitation }\end{array}$ \\
\hline & \multicolumn{8}{|c|}{ One Key } \\
\hline \multirow[t]{2}{*}{$\begin{array}{l}\text { Proportion } \\
\text { RT } \\
\text { \%Error }\end{array}$} & $\begin{array}{c}.2 \\
9.33\end{array}$ & $349^{.4}$ & $319^{.4}$ & +30 & $\begin{array}{c}.4 \\
1.69\end{array}$ & $348^{.4}$ & $340^{.2}$ & +8 \\
\hline & \multicolumn{8}{|c|}{ Two Key } \\
\hline $\begin{array}{l}\text { Proportion } \\
\text { RT } \\
\text { \%Error }\end{array}$ & $\begin{array}{r}442^{.2} \\
3.33\end{array}$ & $414^{.4} .19$ & $\begin{array}{l}396^{.4} \\
1.94\end{array}$ & +18 & $\begin{array}{l}422^{.4} \\
1.00\end{array}$ & $379^{.4} .67$ & $\begin{array}{l}436^{.2} \\
5.50\end{array}$ & -57 \\
\hline
\end{tabular}

Note-Entries give proportions of each stimulus type, mean $R T$ in milliseconds, percentage error, and inhibition (-) or facilitation $(+)$ in milliseconds. Stimulus conditions connected by dashed underlining were associated with the same response. Inhibition/facilitation is given by $R T_{\text {tone }}-R T_{\text {duat }}$ 
of having to suppress a response to light).

In the two-key conditions, as in the one-key conditions, there were more erroneous tone responses to light under high than under low probability of dual stimulation ( $3.33 \%$ vs. $1.00 \%$ ); however, as this difference was not significant, $t(18)=1.80, .05$ $<\mathrm{p}<.10$, these data do not provide strong support for the notion of a criterion shift.

\section{EXPERIMENT 4}

Experiment 1 demonstrated a modality-specific dominance effect (vision dominating audition). Our tentative interpretation of Experiment 3 is that the dominance effect shown in that experiment is not sensory in its locus. It should be noted that there is not necessarily an inconsistency between these conclusions. It is quite possible for tone and light to be processed independently at a sensory level, but for some sort of dominance effect to be generated "downstream," e.g., there may be an innate disposition to respond to visual signals before simultaneous auditory signals.

A remaining problem is that the methodology employed in Experiments 1 and 3 was different: Experiment 3 used the difference in tone RT on dual and choice trials, while Experiment 1 used simply the number of times subjects responded to the tone first or the light first on dual trials. It is possible that the kind of dominance found in Experiment 3 is unrelated to the kind of dominance found in Experiment 1 . Specifically, it is possible that the interference effect found in Experiment 3 is actually an artifact of task complexity and does not reflect visual dominance at all.

In the two-key response conditions of Experiment 3 , the subjects responded to tones with a keypress, to lights with a different keypress, and to dual stimulation with just the "tone" keypress. On dual stimulation trials, the subject must suppress any tendency that might exist to respond to light. By contrast, in the conditions involving the one-key response, the subject is not set to respond to light, and so no response competition should be en- gendered on dual trials. In other words, in the twokey task, dual stimulation trials may be more difficult than tone trials because of implicit response competition.

We tested the modality-specific dominance explanation and the task-difficulty explanation by repeating the two-key low-probability condition of Experiment 3 but with a simple change in the stimulus-response mapping on dual trials. The subjects responded to the tone by pressing the tone key and to the light by pressing the light key, but now, on dual trials, they responded by pressing the light key rather than the tone key. The alternative explanations lead to the following predictions: On the one hand, if "dominance" is due to task difficulty (mediated via response competition), then light RT on dual trials should be significantly slower than light RT on light-only choice trials, since the subject will have to suppress responses to the tonal component of the complex signals. On the other hand, if dominance is modality-specific, as in Experiment 1, then RT on dual trials should be no slower than RT on light trials since light is the dominant signal.

\section{Method}

Ten college students, six male and four female, served as paid subjects. The apparatus, stimuli, and procedure were the same as in Experiment 3. Each subject received $\mathbf{3 0 0}$ test trials; 120 were light alone, 120 were tone alone, and 60 were dual. The subjects were instructed to press the tone key when tone alone occurred, the light key when light alone occurred. In addition, they were to press the light key on dual trials. Thus, this condition is the same as the low-probability two-key condition of Experiment 3 except that the light key instead of the tone key was to be pressed on dual trials. This condition is illustrated in the right half of Table 2; the left half shows a condition described below.

\section{Results and Discussion}

Reaction time. Mean RTs were: light alone, $415 \mathrm{msec}$; tone alone, $444 \mathrm{msec}$; and dual, $422 \mathrm{msec}$ (these data, along with error rates, are also shown in Table 2). The 7-msec difference between light alone and light when accompanied by tone was insignificantly different from zero, $t(9)=1.34$, $\mathrm{p}>.1$. The difference between the (insignificant) 7-msec dominance effect found in this experiment

Table 2

Design and Results of Experiment 4

\begin{tabular}{|c|c|c|c|c|c|c|c|c|}
\hline & \multicolumn{4}{|c|}{ High Probability of Dual Stimulation } & \multicolumn{4}{|c|}{ Low Probability of Dual Stimulation } \\
\hline & Tone & Light & Dual & $\begin{array}{l}\text { Inhibition or } \\
\text { Facilitation }\end{array}$ & Tone & Light & Dual & $\begin{array}{l}\text { Inhibition or } \\
\text { Facilitation }\end{array}$ \\
\hline $\begin{array}{l}\text { Proportion } \\
\text { RT } \\
\text { \%Error }\end{array}$ & $\begin{array}{c}.2 \\
0 \\
0\end{array}$ & $394^{.4} .50$ & $376^{.4} .41$ & +18 & $\begin{array}{c}444^{.4} \\
1.75\end{array}$ & $415^{.4}$ & $\frac{422^{.2}}{2.83}$ & -7 \\
\hline
\end{tabular}

Note-Entries give proportions of each stimulus type, mean $R T$ in milliseconds, percentage error, and inhibition (-) or facilitation ( + ) in milliseconds. Stimulus conditions connected by dashed underlining were associated with the same response. Inhibition/facilitation is given by $R T_{\text {light }}-R T_{\text {duat }}$ 
and the 57-msec dominance effect found in the corresponding condition of Experiment 3 was assessed and found to be significant, $\mathrm{t}(18)=3.56, \mathrm{p}<.01$. This finding suggests that task complexity is not solely responsible for the dominance effect found in Experiment 3. Instead, it reinforces the implication of Experiment 1 that dominance effects are modality specific and it also provides validation for our technique of measuring dominance (i.e., subtracting mean RT to signal when accompanied by accessory stimulus from mean RT on signal alone.)

In Experiment 3, we observed an effect of probability of dual trials. We thought it desirable to check that finding in the case where subjects press the light key on dual trials. Therefore, we ran an additional 10 subjects on a condition comparable to the highprobability two-key condition of Experiment 3. The proportions of light, tone, and dual trials were .4, $.2, .4$, and the subjects responded by pressing the light key on dual trials. This condition resulted in an 18-msec facilitation of the light response, $t(9)$ $=3.15, p<.02$. Moreover, the effect of probability manipulation between conditions in Experiment 4 was significant $(18-\mathrm{msec}$ facilitation vs. $7-\mathrm{msec}$ dominance), $\mathrm{t}(18), \mathrm{p}<.01$. It should be pointed out that, because subjects responded to tone on some trials, the tone was not simply an accessory stimulus. Thus, this condition does not demonstrate intersensory facilitation in the usual sense. Of course, the same comment applies to the high-probability two-key condition of Experiment 3.

Errors. Percentages of erroneous responses for light, tone, and dual trials appear in Table 2.

The "false alarm" rates (here, light responses to tone stimuli) were very low $(0 \%$ and $1.75 \%)$. Moreover, they were in the direction opposite to what would be expected on the basis of the kind of criterion shift that was invoked to describe the pattern of errors in the one-key conditions of Experiment 3. Assuming criterion shifting is a reliable phenomenon, it may be limited to one-key conditions, which are associated with intersensory facilitation.

\section{EXPERIMENT 5}

The visual dominance effect appears to be nonsensory in locus. We have demonstrated this by manipulating nonsensory factors such as stimulusresponse mappings and probability of dual stimulation trials. We suspect that, in part, such manipulations have their effect by changing the distribution of attention to the various signals that may occur. If this is the case, it ought to be possible to obtain a similar result by giving a subject explicit verbal instructions to attend to a particular modality rather than by inducing him to do so by varying the frequency of responses required to that modality.

In the present experiment, we took the condition that has produced the maximum dominance effect in the preceding studies (Experiment 3: two-key, low probability of dual trials) and attempted to reduce, or possibly even eliminate, the dominance effect by an instructional manipulation. In that condition, subjects received tone alone on 120 trials, light alone on 120 trials, and dual stimulation on 60 trials (on which they responded with the tone key). Thus they had to press the tone key on $60 \%$ of all trials. Even though tone responses were required on a majority of trials, a strong visual dominance effect was observed. The present experiment involved two conditions. One was an exact replication of the condition just described. The other was identical in all respects to the first except that subjects were informed that the majority of responses would be to tones and that they should therefore attend to their ears during the experiment.

The design and results of this experiment are shown in Table 3. There was a $51-\mathrm{msec}$ dominance effect in the condition that replicated the highdominance conditions of Experiment $3, \mathrm{t}(9)=4.90$, $\mathrm{p}<.002$. In the "attend to ears" condition, the dominance effect was only $17 \mathrm{msec}, \mathrm{t}(9)=2.40$, $\mathrm{p}<.05$. Most importantly, however, the two dominance effects differed significantly from one another, $\mathrm{t}(18)=2.71, \mathrm{p}<.02$. Thus, we are led

Table 3

Design and Results of Experiment 5

\begin{tabular}{|c|c|c|c|c|c|c|c|c|}
\hline & \multicolumn{4}{|c|}{ Attend to Ears } & \multicolumn{4}{|c|}{ Control } \\
\hline & Light & Tone & Dual & $\begin{array}{l}\text { Inhibition or } \\
\text { Facilitation }\end{array}$ & Light & Tone & Dual & $\begin{array}{l}\text { Inhibition or } \\
\text { Facilitation }\end{array}$ \\
\hline Proportion & .4 & .4 & .2 & & .4 & .4 & .2 & \\
\hline & 499 & 415 & 432 & -17 & 494 & 436 & 487 & -51 \\
\hline \%Error & 2.33 & .58 & 3.33 & & 2.83 & .25 & 6.17 & \\
\hline
\end{tabular}

Note-Entries give proportions of each stimulus type, mean $R T$ in milliseconds, percentage error, and inhibition ( -1 or facilitation ( + ) in milliseconds. Stimulus conditions connected by dashed underlining were associated with the same response. Inhibition/facilitation is given by $R T_{\text {tone }}-R T_{\text {duat }}$ 
to conclude that visual dominance is to some extent under cognitive control.

\section{EXPERIMENT 6}

We have repeatedly found evidence of visual dominance over audition. It is conceivable that the effect is the result of some kind of systematic bias in the initial matching task; subjects may end up with an intensity level for light that is not really as bright as the tone is loud. Alternatively, the initial equation of stimuli may be adequate, but progressive dark adaptation during the course of the experiment may result in the light's having a greater subjective intensity than the tone.

There is little basis for the first of these arguments. As for the latter argument, we have looked for systematic changes in dominance (or facilitation) during the course of our 300-trial sessions, but have failed to find any.

To speak more directly to both points, we conducted an additional experiment in which dominance was assessed at each of three different subjective light-tone intensity ratios $(.5,1,2)$. Twelve new subjects participated in this study, four male and eight female. In the first part of the experiment, each subject went through three matching procedures (as in Experiment 1) to find light intensities that were subjectively one-half of the tone, equal to the tone, and twice the tone. The tone was set at $65 \mathrm{~dB}$ SPL throughout. The order of the ratios to be matched was balanced across subjects. The resulting luminances for each light-tone ratio were: 17.1, 27.4, and $34.3 \mathrm{~cd} / \mathrm{m}^{2}$.

Following the matching procedure, the subjects served in a RT task, receiving one block of 100 trials at each of the three intensity ratios (again, order of blocks balanced over subjects). The condition selected was the low-probability two-key condition that had resulted in a $57-\mathrm{msec}$ visual dominance effect in Experiment 3 and a 51 -msec visual dominance effect in Experiment 4.

Mean RTs, dominance effects, and error rates appear in Table 4. The dominance effects were significant for all three light-tone ratios: for $.5, \mathrm{t}(11)$ $=2.52, \mathrm{p}<.05$; for $1, \mathrm{t}(11)=3.53, \mathrm{p}<.01$; and for $2, t(11)=3.60, p<.01$. An overall analysis of variance of the dominance effects showed that the differences among the three experimental conditions (i.e., the subjective intensity ratios) were not significant, $\mathbf{F}(2,22)<1$.

\section{GENERAL DISCUSSION}

Visual dominance over audition appears under several modes of testing. In Colavita's experiments, subjects were not specifically instructed to respond to both signals on dual trials and dominance showed up as an apparent inability to respond to tone when a light was simultaneously present. Indeed, subjects were sometimes unaware that a tone had been presented on dual trials. In our first experiment, when subjects were instructed to respond to both tone and light on dual trials, we obtained no evidence of unawareness of or inability to respond to sound. However, visual dominance did manifest itself, in this case as a tendency to respond to the light more quickly than to the tone.

As important as the appearance of visual dominance was the discovery of manipulations that reduced, eliminated or even reversed it. Three potent factors are the necessity of responding to the light signal, the probability of the three trial types (light, tone, dual), and instructional set. Taken together, these factors suggest that visual dominance is not a phenomenon that depends on the "hard-wiring" of the nervous system. Instead, these findings implicate a system that is under at least partial cognitive control.

We think it is also important to note that relative intensity of the tone and light did not affect the magnitude of visual dominance. This observation is congruent with the hypothesis that visual dominance arises at processing stages subsequent to stimulus encoding (cf. Sternberg, 1967).

Our data are consistent with what has been called the independent channels hypothesis. According to this hypothesis, as elaborated by Egeth (1977) and Shiffrin (e.g., Shiffrin \& Grantham, 1974) among others, early sensory processing has the following characteristics: (1) several inputs can be processed simultaneously without mutual interference, (2) an input receives the same perceptual analysis regardless of whether or not it is "attended," and

Table 4

Design and Results of Experiment 6

\begin{tabular}{|c|c|c|c|c|}
\hline $\begin{array}{l}\text { Light/Tone } \\
\text { Ratio }\end{array}$ & Light & Tone & Dual & $\begin{array}{l}\text { Inhibition or } \\
\text { Facilitation }\end{array}$ \\
\hline $1^{.5}$ & $\begin{array}{l}453(1.05) \\
438(1.88) \\
452(1.88)\end{array}$ & $\begin{array}{l}370(.42) \\
382(1.05) \\
384(.42)\end{array}$ & $\begin{array}{l}410(7.10) \\
432(5.85) \\
433(6.25)\end{array}$ & $\begin{array}{l}-40 \\
-50 \\
-49\end{array}$ \\
\hline
\end{tabular}

Note-Entries give mean RT in milliseconds, percentage error in parentheses, and inhibition $(-)$ or facilitation (+) in milliseconds. Stimulus conditions connected by dashed underlining are associated with the same response. Inhibition/facilitation is given by $R T_{\text {tone }}-R T_{\text {duat }}$ 
(3) capacity limitations in processing are due to stages subsequent to encoding.

Intersensory facilitation can be explained in terms consistent with the independent channels hypothesis. Nickerson (1973) argued that the occurrence of a stimulus can have one of two effects: it can evoke a response or it can cause the subject to increase his preparedness to respond. (Which of these effects a stimulus will have is assumed to be under the subject's control.) In the classical intersensory facilitation paradigm, the occurrence of the accessory stimulus has the effect of increasing the subject's preparedness to respond to the target stimulus.

The obvious problem is to explain how one stimulus can increase the preparedness to respond to another if the two are presented simultaneously, as was the case in the one-key conditions of the present experiments. Nickerson handled this by recourse to the now familiar notion of a "horserace" between the two signals. The central arrival-time distributions for such stimuli would likely overlap to a considerable extent. Thus, on some trials, the accessory stimulus would arrive enough before the target stimulus to produce some enhancement of preparedness. ("Arrival time"' here refers to the total time required for the nervous system to do everything short of actually evoking a response.)

In contrast to intersensory facilitation, visual dominance seems to be restricted to situations in which subjects are responsible for monitoring and responding to two signals within a block of trials. Moreover, visual dominance appears to increase with the amount of attention the subject pays to light. Thus, in Experiment 5, when subjects were told explicitly to attend to their ears, visual dominance was reduced. In Experiment 3 (two-key condition), dominance was greater when the probability of making a response to light was .4 than when it was .2 . Indeed, in the latter condition, there was a reverseddominance rather than a dominance effect. Similar comments can be made about Experiment 4, where, again, a reversed-dominance effect was observed with high probability of dual stimulation.

In Experiment 4, where subjects responded to light on dual trials, as the probability of responding to tone increased from .2 to .4 , facilitation of light responses by tone gave way to (nonsignificant) dominance of tone over light. It is interesting to speculate that if a sufficiently high proportion of tone responses were required, perhaps in conjunction with "attend to ears" instructions, it might be possible to demonstrate a significant auditory dominance effect. This speculation, as well as the major findings of this study, are generally consistent with the recent explanation of visual dominance in terms of attentional mechanisms offered by Posner et al. (1976). Vision ordinarily dominates audition (and cutaneous sensitivity as well) because, for still unknown reasons, subjects are disposed to attend to vision, everything else being equal. The various manipulations of the present experiment may be seen as ways of making everything else not equal, which results in changes in the distribution of attention, which, in turn, results in variation in the magnitude of "sensory" interaction.

\section{Relations to Other Work}

Visual dominance is closely related to a wide variety of other topics in human experimental psychology. While it is beyond the range of this paper to pursue all of these connections, it does seem worthwhile to discuss a few of the most relevant ones.

Central intermittency. Kristofferson (1967) has presented evidence that attention can be thought of as the result of a gating mechanism that controls the flow of information from sensory channels into a central processor. According to this model, the gating is an all-or-none operation; the central processor can be open to only one channel at any time. It is further hypothesized that the temporal integration of data processing activities is achieved through the control of an internal "clock" which generates a succession of equally spaced points in time. These time points determine when attention can, but need not, switch from one channel to another, and also when the signals in one stage of central processing can be transmitted into a subsequent stage.

When attention is aligned with a particular channel, then messages arriving on that channel may be transmitted into the central processor at the next time point. When messages arrive on an unattended channel, however, attention must first be switched to that channel at one time point, and then the message may enter the central processor at the following time point. Central processing of unattended messages, therefore, will be delayed by the time required to switch attention. Note that this model necessitates the assumption that an unattended input can signal the gating mechanism to switch attention.

The visual dominance effects of the present study are consistent with this model of attention. When subjects were most likely to be attending to their ears, as in the one-key conditions of Experiments 2 and 3 , responses to the tone were not delayed by the simultaneous occurrence of the light. When experimental conditions favored a bias toward attending to the eyes (e.g., Experiment 6), however, the presence of a light did cause a delay in the tone response. This delay could be explained as follows: between trials, subjects are attending to their eyes. When a tone occurs alone, attention must be switched, resulting 
in a processing delay of one time unit. When a tone and light occur simultaneously, attention remains aligned with the visual channel until the light enters the central processor, and then attention is switched to the auditory channel. This results in a processing delay of at least two time units for the tone, since the tone cannot enter the central processor until the light has exited.

Prior entry. When the subject is presumably attending to the visual channel, his response to a tone appears to be delayed. It also appears that, under such conditions, his perception of the temporal order of a simultaneous light and tone will be biased in favor of judging the light to have occurred before the tone. This is simply a restatement of the law of prior entry, which was included by Titchener (1908, p. 251) among his seven laws of attention. Experimental evidence in support of prior entry has been provided by Sternberg, Knoll, and Gates (Note 1), who confirmed that temporal order judgments can be biased through instructions to attend to a particular modality. It is also of particular interest in the present context that this attentional biasing effect appears to be nonsensory in locus (Sternberg \& Knoll, 1973; Sternberg et al., Note 1), in agreement with Colavita's and our finding that visual dominance is not affected by manipulations of stimulus intensity.

It is tempting to speculate that the visual dominance effects of the present study could also be manifested as a bias in temporal order judgments of a light and tone. Comparisons between reaction times and temporal order judgments should be interpreted with caution, however, as Sternberg and Knoll (1973) have discussed. A study of Vanderhaeghen and Bertelson (1974) has substantiated this warning. On each trial, a reference tactile stimulus and another stimulus, which was unpredictably either a sound or a light, were presented with variable onset times. The subject's task was to make a fast choice reaction (sound or light) and to judge the temporal order of the two stimuli. Manipulations of the relative frequency of occurrence of the uncertain stimulus affected RTs as predicted (RTs were faster to the more frequent stimulus) but produced unsystematic effects on apparent order.

At least one failure to find a consistent relationship between RTs and temporal order judgments, however, can be handled by the notion of visual dominance over audition. Rutschmann and Link (1964) measured simple RTs to visual and auditory stimuli, and also measured judgments of temporal order as a function of the delay between onsets of these stimuli. Their results appear contradictory: RTs were faster to the auditory stimulus, yet the onset of the auditory stimulus had to precede the visual stimulus in order for the two to be judged as simultaneous. This apparent discrepancy can be resolved, however, with the assumption that, in the absence of specific instructions or incentives to the contrary, subjects were biased in favor of attending first to their eyes and then to their ears during the temporal order judgments, but attended only to the relevant modality during the simple RT trials. The shorter latency for processing auditory information which was observed during RT trials lost its advantage over light when an additional delay for attention switching was added to processing time.

\section{REFERENCE NOTE}

1. Sternberg, S., Knoll, R. L., \& Gates, B. Prior entry reexamined: Effects of attentional bias on order perception. Paper presented at Annual Meeting of the Psychonomic Society, St. Louis, 1971.

\section{REFERENCES}

Békésy, G. von. Mach- and Hering-type lateral inhibition in vision. Vision Research, 1968, 8, 1483-1499.

Bender, M. B. Perceptual interactions. In D. Williams (Ed.), Modern trends in neurology. London: Butterworth, 1970.

Bernstein, I. H., Chu, P. K., Briggs, P., \& Shurman, D. L. Stimulus intensity and foreperiod effects in intersensory facilitation. Quarterly Journal of Experimental Psychology. 1973, 25, 171-181.

Colavita, F. B. Human sensory dominance. Perception \& Psychophysics, 1974, 16, 409-412.

Cornsweet, T. N. Visual perception. New York: Academic Press, 1970.

Donders, F. C. Die Schnelligkeit psychischer Processe. Archiv für Anatomie und Physiologie, 1868, 657-681.

Egeth, H. E., \& Pachella, R. Multidimensional stimulus identification. Perception \& Psychophysics, 1969, 5, 341-346.

EgETh, H. Attention and preattention. In G. H. Bower (Ed.), The psychology of learning and motivation (Vol. 11). New York: Academic Press, 1977.

EIJKMAN, E., \& VENDRIK, A. J. H. Can a sensory system be specified by its internal noise? Journal of the Acoustical Society of America, 1965, 37, 1102-1109.

GIlliom, J. D., \& Sorkin, R. D. Sequential vs. simultaneous two-channel signal detection: More evidence for a high-level interrupt theory. Journal of the Acoustical Society of America, 1974, 56, 157-164.

KoHfeld, D. L. Simple reaction time as a function of stimulus intensity in decibels of light and sound. Journal of Experimental Psychology, 1971, 88, 251-257.

KRistofferson, A. B. Attention and psychophysical time. Acta Psychologica, 1967, 27, 93-100.

Lindsay, P. H. Multichannel processing in perception. In D. I. Mostofsky (Ed.). Attention: Contemporary theory and analysis. New York: Appleton-Century-Crofts, 1970.

Massaro, D. W., \& KAHN, B. J. Effects of central processing on auditory recognition. Joumal of Experimental Psychology, 1973, 97, 51-58.

Melzack, R., \& WAll, P. D. Pain mechanisms: A new theory. Science, 1965, 150, 971-979.

Moore, J. J., \& Massaro, D. W. Attention and processing capacity in auditory recognition. Joumal of Experimental Psychology, 1973, 99, 49-54.

Nickerson, R. S. Intersensory facilitation of reaction time. Psychological Review, 1973, 80, 489-509.

Posner, M. I., Nissen, M. J., \& KLeIN, R. M. Visual dominance: 
An information-processing account of its origins and significance. Psychological Review, 1976, 83, 157-171.

Rutschmann. J., \& Link, R. Perception of temporal order of stimuli differing in sense mode and simple reaction time. Perceptual and Motor Skills, 1964, 18. 345-352.

Shiffrin, R. M., \& Grantham. D. W. Can attention be allocated to sensory modalities? Perception \& Psychophysics, 1974, 15, 460-474.

STERnberg, S. Two operations in character recognition: Some evidence from reaction-time measurements. Perception \& Psychophysics, 1967. 2. 45-53.

STERNBERG, S. \& Kroll. R. L. The perception of temporal order: Fundamental issues and a general model. In $\mathbf{S}$. Kornblum (Ed.), Attention and performance IV. New York: Academic Press. 1973.

Titchener. E. B. Lectures on the elementary psychology of feeling and attention. New York: Macmillan, 1908.

Tulving, E., \& Lindsay, P. H. Identification of simultaneously presented simple visual and auditory stimuli. In A. F. Sanders (Ed.), Attention and performance. Amsterdam: North-Holland, 1967.

Vanderhaeguen, C., \& Bertelson, P. The limits of prior entry: Nonsensitivity of temporal order judgments to selective preparation affecting choice reaction time. Bulletin of the Psychonomic Society, 1974, 4. 569-572.

WoOdWorth, R. S., \& SCHxosberg, H. Experimental psychology. New York: Holt. 1954.

\section{NOTES}

1. Note that in Colavita's experiment the first key pressed turned off both stimuli on dual stimulation trials, whereas in the present experiment each key turned off only the corresponding stimulus. We made this change simply to force two responses on each dual trial. Of course, it is not reasonable to expect to find any subjects being unaware of one of the stimuli under these circumstances.

2. We do not attach great weight to this statistical test, as it is based on small (and unequal) numbers of responses across subjects. In fact, the data from one subject had to be discarded because he made no tone responses first on dual trials.

3. Note that while simple auditory RT is often found to be faster than simple visual RT (e.g., Woodworth \& Schlosberg, 1954), this is not inevitable. In particular, Kohfeld (1971) found that tones and lights matched in terms of decibels above their respective absolute threshold yielded approximately equal RTs at least for photopic visual levels. The important point is that, under our particular experimental conditions, it was the stimulus that yielded the slower simple RT (light) that dominated in the dual stimulation task.

4. Since the probabilities of light, tone, and dual trials must sum to 1.0 , the probability of dual trials is confounded here with probability of light-only trials. We leave to later research the full determination of the relative importance of the various probability relations in this design.
(Received for publication December 18, 1976; revision accepted May 3, 1977.) 